\title{
Deletion of low-density lipoprotein-related receptor 5 inhibits liver Cancer cell proliferation via destabilizing Nucleoporin 37
}

Jinxiao Chen ${ }^{1 \dagger}$, Da Wo $^{1 \dagger}$, En Ma ${ }^{3}$, Hongwei Yan ${ }^{3}$, Jun Peng ${ }^{2}$, Weidong Zhu ${ }^{3}$, Yong Fang ${ }^{1 *}$ and Dan-ni Ren ${ }^{2^{*}}$ (D)

\begin{abstract}
Background: LRP5/6 are co-receptors in Wnt/ $\beta$-catenin pathway. Recently, we discovered multiple $\beta$-catenin independent functions of LRP5/6 in tumor cells and in the diseased heart. Nucleoporin 37 (NUP37) is an important component of the nuclear pore complex (NPC), whose elevated expression is associated with worsened prognosis in liver cancer. Previous studies have shown that NUP37 interacted with YAP and activated YAP/TEAD signaling in liver cancer. Our preliminary findings showed a nuclear location of LRP5. We thus tested the hypothesis that LRP5 may act as a genuine regulator of YAP/TEAD signaling via modulating NUP37 in a $\beta$-catenin-independent way.
\end{abstract}

Methods: We performed siRNA knockdown of LRP5, LRP6, or $\beta$-catenin in liver cancer HepG2 cells to determine the effect on tumor cell proliferation. Protein expressions and interaction between LRP5 and NUP37 were determined using immunoprecipitation and western blot analyses.

Results: HepG2 cell proliferation was markedly inhibited by knockdown of LRP5 but not LRP6 or $\beta$-catenin, suggesting that LRP5 has a specific, $\beta$-catenin-independent role in inhibiting HepG2 cell proliferation. Knockdown of NUP37 by siRNA inhibited the proliferation of HepG2 cells, whereas overexpression of NUP37 reversed the decrease in cell proliferation induced by LRP5 knockdown. Immunoprecipitation assays confirmed that LRP5 bound to NUP37. Furthermore, LRP5 overexpression restored NUP37 knockdown-induced downregulation of YAP/TEAD pathway.

Conclusions: LRP5 deletion attenuates cell proliferation via destabilization of NUP37, in a $\beta$-catenin-independent manner. LRP5 therefore acts as a genuine regulator of YAP/TEAD signaling via maintaining the integrity of the NPC, and implicates a therapeutic strategy in targeting LRP5 for inhibiting liver cancer cell proliferation.

Keywords: LRP5, NUP37, Nuclear pore complex, Wnt/ß-catenin signaling, Cancer cell proliferation

\section{Background}

Low-density lipoprotein-related receptors 5 and 6 (LRP5/6) are commonly regarded as Wnt coreceptors involved in activating $W n t / \beta$-catenin pathway [1-3]. Upon binding to

\footnotetext{
* Correspondence: fangyong1020@hotmail.com; danny1217@163.com † Jinxiao Chen and Da Wo contributed equally to this work.

'Department of Plastic and Burn Surgery, Ninth People's Hospital, Shanghai Jiaotong University, School of Medicine, 639 Zhi Zao Ju Road, Shanghai 200011, People's Republic of China

${ }^{2}$ Fujian Key Laboratory of Integrative Medicine on Geriatric, Academy of Integrative Medicine, Fujian University of Traditional Chinese Medicine, 1 Qiuyang Road, Minhou Shangjie, Fuzhou 350122, Fujian, China Full list of author information is available at the end of the article
}

Wnt ligands, LRP5/6 cooperates with Frizzled to activate Wnt $/ \beta$-catenin signaling pathway and subsequently prevent the ubiquitination and degradation of cytoplasmic $\beta$ catenin, thereby leading to the nuclear translocation of $\beta$ catenin and activation of Wnt target genes [4-6]. Recently, we reported that LRP5/6 could prevent Frizzled-regulated non-canonical pathway activation via directly binding to the Frizzled receptor [7], and established a novel working model on the roles of LRP5/6 in canonical and noncanonical pathways. Furthermore, we showed that Wnt inhibitors insulin-like growth factor binding protein 4 (IGFBP-4) and Dickkopf-1 (DKK1) played opposing roles 
in cardiac ischemia via differential targeting to LRP5/6 and $\beta$-catenin [8]. A separate study showed that LRP6 but not LRP5 deletion greatly promoted mTOR phosphorylation and acted as a major regulator of cardiomyocyte cell growth in a $\beta$-catenin-independent manner [9]. These studies demonstrated that LRP5/6 have various Wnt/ $\beta$-catenin-independent physical and pathological functions that are important during adult homeostasis. However, the biological diversity and roles of LRP5/6 are yet to be fully elucidated.

The nuclear pore complex (NPC) is composed of roughly 34 different proteins termed nucleoporins (NUPs) that assemble together to form a large $\sim 120$ megadalton transport channel embedded in the nuclear envelope. Maintaining the integrity of the NPC is critical, which would otherwise impact the regulation and shuttling of numerous signaling proteins [10, 11]. Nucleoporin 37 (NUP37) is an indispensable component of the conserved NUP107-160 complex, which locates in the outer rings of the NPC and composes a major scaffold module of the NPC assembly [12, 13]. Recent studies have shown that upregulated expression of NUP37 in HCC acts as a positive regulator of YAP/ TEAD signaling, thereby promoting cancer progression [14]. In addition, data from Human Protein Atlas showed that there was a negative correlation between the expression of NUP37 and patient survival rate [15]. Therefore NUP37 is regarded as a prognostic indicator for liver cancer, where high expression of NUP37 is associated with worsened patient prognosis.

HepG2 cells harbor a constitutively active mutant of $\beta$-catenin, which results in the over-activation of Wnt $/ \beta$ catenin signaling [16]. Thus, we utilized HepG2 cells as an ideal model for studying the $\beta$-catenin-independent functions of LRP5/6. Here, we examined the effect of LRP5, LRP6, or $\beta$-catenin knockdown on liver cancer HepG2 cell proliferation. Previous studies have shown that NUP37 interacted with YAP and activated YAP/ TEAD signaling by enhancing the interaction between NUP37 and YAP [14]. Accordingly, we explored the relationship between LRP5/6 and NUP37, and its effect on YAP/TEAD signaling. Our findings provide important insights into the roles of LRP5 and NUP37 in maintaining the integrity of the NPC and subsequent promotion of cancer progression in hepatocellular carcinoma (HCC). Taken together, we revealed the $\beta$-cateninindependent biological functions of LRP5 in inhibiting the proliferation of HepG2 cells.

\section{Materials and methods}

\section{Cell proliferation and siRNA knockout assay}

HepG2 cells were seeded on $35 \mathrm{~mm}$ dishes using Dulbecco's modified Eagle's medium (DMEM, Gibco, USA) and cultured at $37^{\circ} \mathrm{C}$ in an incubator containing $5 \% \mathrm{CO}^{2}$. Cells were transfected using RNAiMAX (Invitrogen, Carlsbad, CA, USA) with siRNAs for human LRP5 (HSS106156-1\#, HSS106157-2\#, HSS106158-3\#), human LRP6 (HSS 106153), human $\beta$-catenin (HSS102460), human NUP37 (HSS128152-a\#, HSS128153-b\#, HSS128154-c\#) or negative control siRNA (Invitrogen) in OPTI-MEM (Invitrogen). Cells were counted and imaged $48 \mathrm{~h}$ after transfection for further analysis. Results are representative of at least three independent experiments.

\section{MTT and CCK8 assay}

MTT and CCK8 cell proliferation and cell viability assays were performed to measure the viability and proliferation of HepG2 cells, according to the manufacturer's instructions (MTT, C0009, Beyotime Biotechnology) and (CCK8, C0038, Beyotime Biotechnology). Briefly, cells were knock down by siRNAs after $48 \mathrm{~h}$, then seeded in 96 well plates and cultured for another $72 \mathrm{~h}$. MTT or CCK8 solution were then added to each well and incubated at $37^{\circ} \mathrm{C}$ for $4 \mathrm{~h}$ for MTT, or $1 \mathrm{~h}$ for CCK8, prior absorbance measurements at $570 \mathrm{~nm}$ for MTT, or 450 $\mathrm{nm}$ for CCK8, using a microplate reader (SpectraMax ${ }^{\circ}$ M5 Microplate Reader, Molecular Devices).

\section{Western blotting assay}

Western blotting was performed as previously described [17]. Briefly, total protein was extracted using RIPA buffer (Beyotime Biotechnology), resolved on SDS-PAGE gels and transferred onto PVDF membranes. Following blocking with non fat dry milk in TBST, membranes were washed and incubated with primary antibodies for (anti-LRP5, \#5731, CST; NUP37, ab220675, Abcam; anti-CTGF, \#86641, CST; Flag-tag antibody and myc-tag antibody from Genscript) overnight at $4{ }^{\circ} \mathrm{C}$. Membranes were then incubated with the appropriate HRP-conjugated secondary antibodies for $1 \mathrm{~h}$ at room temperature and detected with chemiluminescence using Immobilon Chemiluminescent HRP Substrate (Millipore). Results are representative of at least three independent experiments.

\section{Real-time PCR assay}

Total RNA was extracted using TRIzol reagent (Takara Biotechnology, China) and reverse-transcribed to cDNA using a Prime Script II cDNA Synthesis Kit (Takara Biotechnology) according to the manufacturer's instructions. Real-time quantitative PCR was performed with SYBR-Green master mix (Applied Biosystems, Foster City, CA, USA) in 96-well optical plates using a QuantStudio 6 Flex Real-Time PCR System (Thermo Fisher Scientific). GAPDH was used as the reference gene for determination of relative gene expressions. Results are representative of at least three independent experiments. 


\section{Co-immunoprecipitation assay}

Co-immunoprecipitation (co-IP) analyses were performed as previously described. Briefly, plasmids were transiently transfected into HEK293 cells for $48 \mathrm{~h}$. Cells were lysed using NP-40 lysis buffer (Beyotime) and IP was performed using Anti-Flag M2 Affinity Gel (Sigma), or anti-c-Myc Agarose (Thermo Fisher Scientific) and mouse IgG Agarose as a negative control (Thermo Fisher Scientific). After incubation at $4{ }^{\circ} \mathrm{C}$ for $2 \mathrm{~h}$, agarose was washed three times with TBST, then incubated with $100 \mu \mathrm{l} 0.1 \mathrm{~N} \mathrm{NH}_{4} \mathrm{OH}$ for $5 \mathrm{~min}$. Following centrifugation, the resulting supernatant was collected and neutralized to $\mathrm{pH} 7.0$ with using acetic acid. The samples were heated in $1 \times$ reducing loading buffer at $95^{\circ} \mathrm{C}$ for 5 min and subsequent Western Blot analysis was performed to examine protein binding. Results are representative of at least three independent experiments.

\section{Tumor implantation assay}

All animal protocols were approved by the Animal Care and Use Committee of Fujian University of Traditional
Chinese Medicine and followed the ARRIVE guidelines. LRP5/6 siRNAs, $\beta$-catenin siRNA, or control siRNA were transfected into HepG2 cells using RNAiMAX. After $48 \mathrm{~h}$, a total of $1 \times 10^{6}$ cells were subcutaneously injected into the lower flanks of severe combined immunodeficient/beige (SCID/bg) mice. Tumor diameters were measured with digital calipers and the tumour volume in $\mathrm{mm}^{3}$ was calculated using the formula: tumor volume $=(\text { width })^{2} \times($ length $/ 2)$.

\section{Reporter gene assay}

Luciferase activity was examined using Luciferase Assay kit (Promega, E1980). Briefly, TOPFLASH reporter gene together with siLRP5/6 or Wnt3a construct was transfected in HEK-293 cells. c-fos/ $\alpha$-MHC-Luciferase reporter gene was transfected in HEK-293/AT1-293 cells following Angiotensin II stimulation after $24 \mathrm{~h}$ transfection using Fugene (Roche) in 48-well plates $\left(3 \times 10^{4}\right.$ cells per well) for $48 \mathrm{~h}$. Lysates of HEK293 cells were measured according to the manufacturer's instructions. All data were normalized by Renilla Luciferase activity.
A
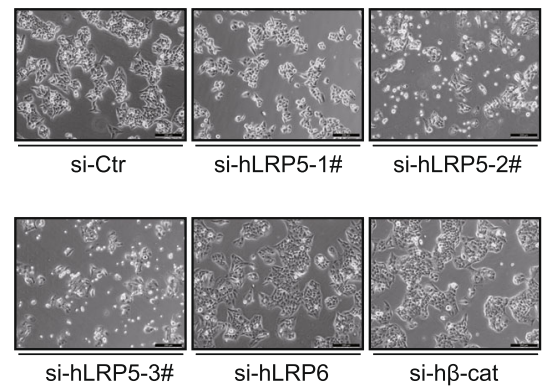

B

CCK8 assay

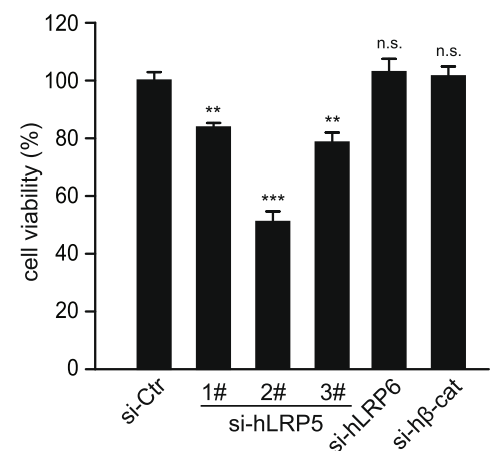

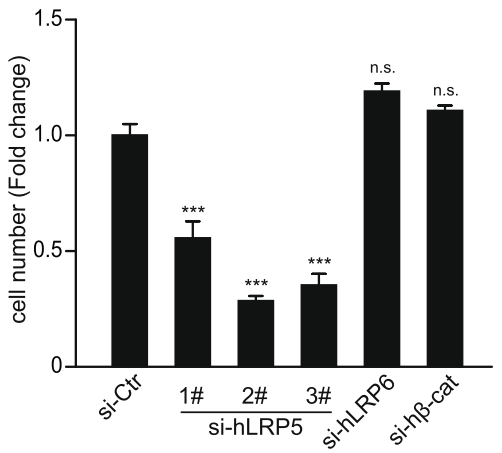

MTT assay

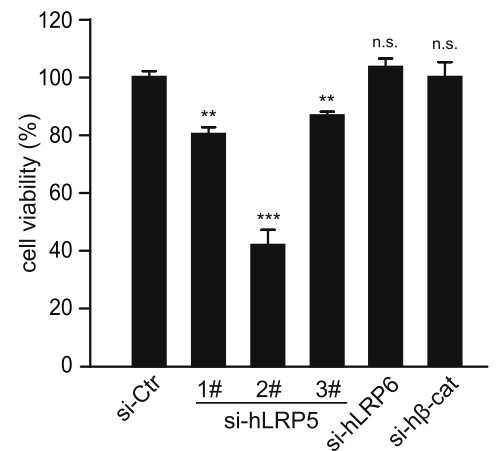

Fig. 1 Knockdown of LRP5 inhibits the proliferation of HepG2 cells. a Representative image of HepG2 cells following siRNA knockdown of LRP5 (three distinct siRNA targeting different segment of LRP5 ORF), LRP6 or $\beta$-catenin for $48 \mathrm{~h}$ (left). $n=3$. Quantification of total cell count. *** $P<$ 0.001, n.s., no significance, compared to siRNA control cells (right). b CCK8 and MTT assays of cell proliferation and cell viability in HepG2 cells following siRNA knockdown of three distinct siRNA targeting LRP5 ORF. ${ }^{* *} P<0.01$, *** $P<0.001$, n.S., no significance, compared to siRNA control cells 


\section{Statistical analysis}

All data were obtained from at least three independent experiments performed in duplicate. Data are presented as the mean \pm SE of the mean (SEM). Graphs and statistical analysis were done using GraphPad Prism (version 6.01; GraphPad Software, Inc). All statistical analyses were analyzed using SPSS 16.0 software (SPSS, Inc., Chicago, IL, USA). P-values of less than 0.05 were considered statistically significant.

\section{Results}

\section{Knockdown of LRP5 inhibits the proliferation of HepG2 cells}

HepG2 cells harbor a constitutive active mutant of $\beta$ catenin with basal over-activation of $\mathrm{Wnt} / \beta$-catenin pathway, which was verified using TOPflash reporter gene assay, which was upregulated 135-fold compared to HEK293 cells, a separate cell line without the active mutant of $\beta$-catenin (Additional file 1: Figure S1). We next examined the tumor formation and Wnt activity following siRNA knockdown of LRP5, LRP6, and $\beta$-catenin. SCID/ bg nude mice injected with HepG2 cells following knockdown of $\beta$-catenin by siRNA had no effect on tumor formation, whereas knockdown of LRP5/6 led to significantly smaller tumor volume (Additional file 1: Figure S2), suggesting a $\beta$-catenin-independent role of LRP5/6 in regulating HepG2 cell proliferation. We further investigated the individual role of LRP5 and LRP6 using TOPflash reporter gene assay in HepG2 cells, which showed that Wnt activity was significantly affected by siRNA knockdown of LRP6, but not LRP5 in the absence or presence of Wnt3a (Additional file 1: Figure S3). Thus, we utilized HepG2 cells as an ideal model for studying the $\beta$-cateninindependent functions of LRP5.

LRP5 and LRP6 are plasma membrane receptors commonly thought to be involved in the activation of Wnt/ $\beta$-catenin pathway. However, the role of LRP5 and LRP6 in liver cancer has not been fully investigated. We used
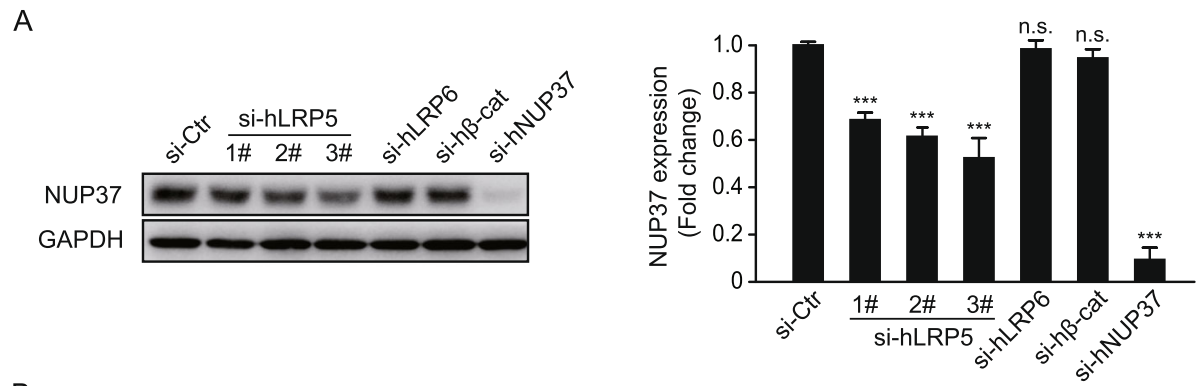

B
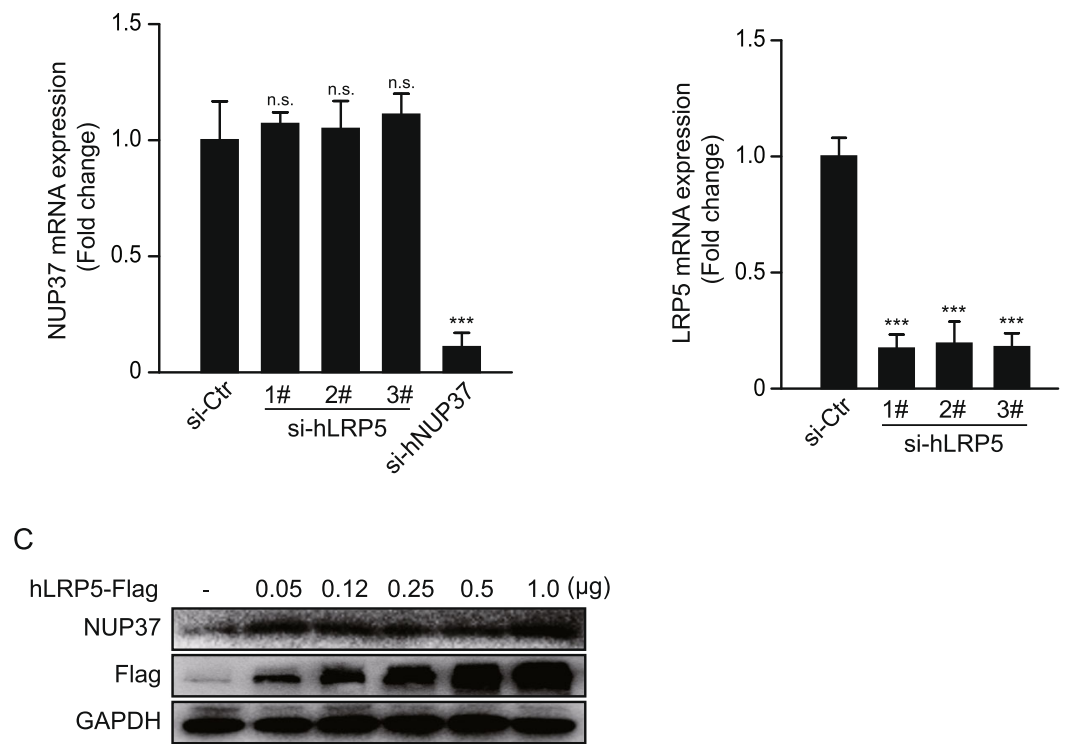

Fig. 2 Knockdown of LRP5 destabilizes NUP37. a Western blot showing the expression of NUP37 following siRNA knockdown of LRP5 (three distinct siRNA targeting different segment of LRP5 ORF) or LRP6 or $\beta$-catenin for $48 \mathrm{~h}$ (left). si-hNUP37, positive control. $n=3$. Densitometry analysis normalized with the loading control, GAPDH (right). b Real-time PCR analysis following siRNA knockdown of LRP5 (three distinct siRNA targeting different segment of LRP5 ORF), showing no significant changes in mRNA expression of NUP37 (left), si-hNUP37, positive control. $n=3$. and significant down-regulation of LRP5 mRNA expression (right). $n=3$. $\mathbf{c}$ Western blot analysis demonstrating that over-expression of LRP5 promotes stabilization of nuclear NUP37 in dose dependent manner, even at relatively low dosage. GAPDH, loading control 
RNA interference method with three distinct (siRNAs) in order to eliminate any ubiquitous off-target effects. Knockdown efficiency of all tested siRNAs, including LRP5, LRP6, $\beta$-catenin, and NUP37 were presented in Additional file 1: Figure S4 and S5. Knockdown of LRP5 with three distinct siRNAs (siLRP5-1\#, siLRP5-2\#, and siLRP5-3\#) all significantly inhibited the proliferation of HepG2 cells, as well as a separate liver cancer cell line Huh7 cells (Fig. 1a and Additional file 1: Figure S6), indicating that knockdown of LRP5 may generally affect the proliferation of liver cancer cells. However, knockdown of LRP6 as well as $\beta$-catenin, the key effector protein in Wnt/ $\beta$-catenin pathway had no obvious effects on HepG2 cell proliferation (Fig. 1a). Furthermore, MTT assay and CCK8 assay were performed to determine the effects of LRP5/6 knockdown on cancer cell viability and proliferation, which showed that knockdown with LRP5, but not LRP6 or $\beta$-catenin, significantly inhibited the cell viability and proliferation of HepG2 cells (Fig. 1b). These results indicated that LRP5 specifically inhibits HepG2 cell viability and proliferation, and furthermore, this effect is independent of $\mathrm{Wnt} / \beta$-catenin pathway.

\section{Knockdown of LRP5 destabilizes NUP37}

Knockdown of LRP5 markedly downregulated the expression of NUP37, but had no effect on its transcription level following treatment with three distinct siRNA targeted open reading frame (ORF) of LRP5, which indicated that the downregulation of NUP37 expression induced by knockdown of LRP5 occurs during the posttranslational modification stage (Fig. 2a and $\mathrm{b})$. Furthermore, overexpression of LRP5 promoted the stabilization of nuclear NUP37, in a dose dependent manner (Fig. 2c).

\section{NUP37 interacts with LRP5 and promotes proliferation of $\mathrm{HCC}$}

Shuttling of signaling proteins between the cytoplasm and nucleus is tightly regulated by the NPC, which is composed of approximately 34 different NUPs that are packaged into a complex cylindrical structure. Previous studies have demonstrated that NUP37 is significantly upregulated in HCC clinical samples, and overexpression of NUP37 can promote proliferation of HCC cells via interacting with YAP and subsequently activating YAP/ TEAD signaling [14]. Here, we further examined the effect on cell proliferation following knockdown of NUP37 in HepG2 cells. Indeed, similar to knockdown of LRP5, knockdown of NUP37 significantly inhibited the proliferation of HepG2 cells (Fig. 3a-c) and Huh7 cells (Additional file 1: Figure S6), as well as a non liver cancer cell line HEK-293 cells (Additional file 1: Figure S7). Interestingly, co-IP analysis demonstrated a strong binding affinity between LRP5 and NUP37 (Fig. 3c), suggesting

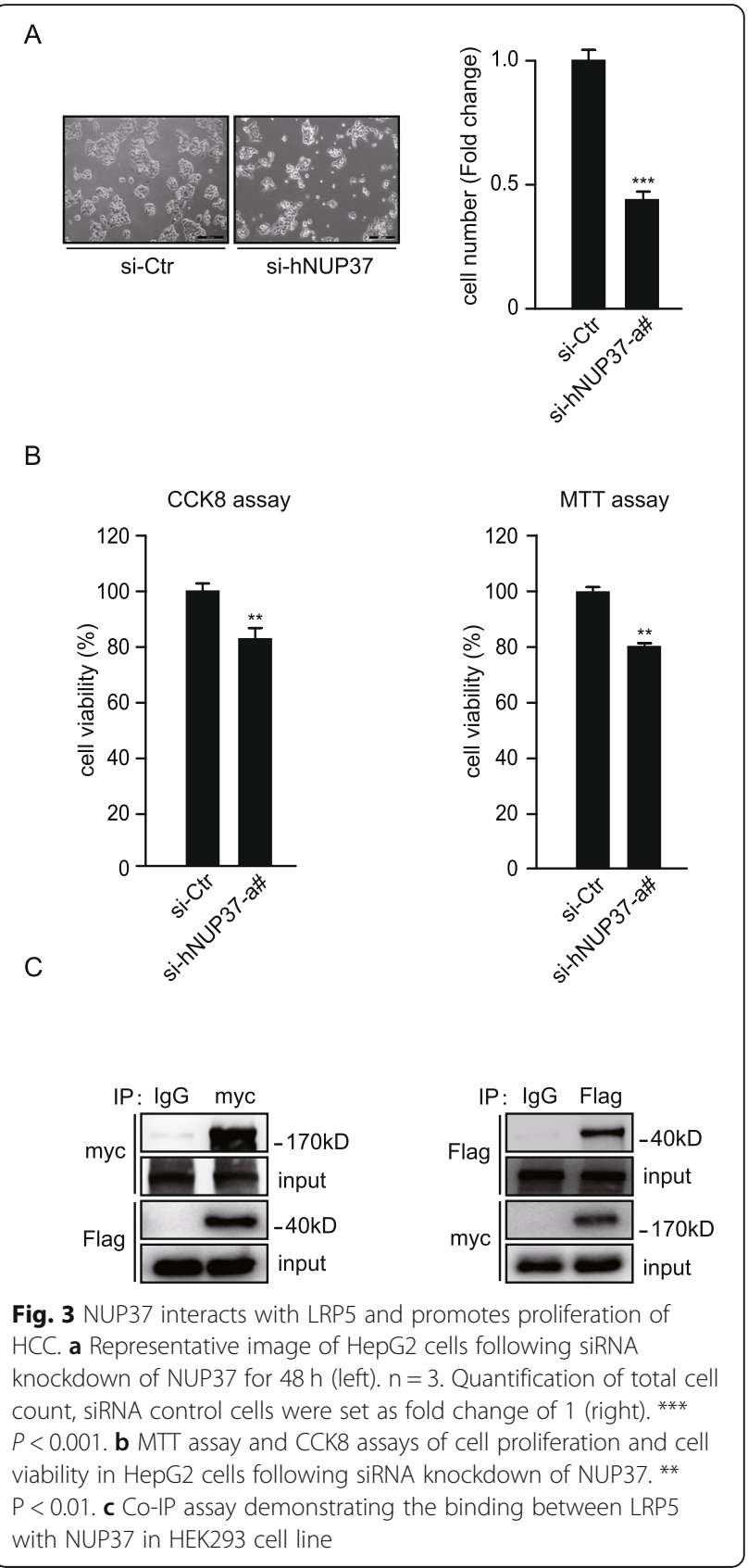

the presence of a molecular regulatory mechanism between LRP5 and NUP37.

In order to verify the fact that knockdown of LRP5 inhibited HepG2 cell proliferation via destabilization of NUP37, we examined whether overexpression of NUP37 could reverse this phenotype. Indeed, overexpression of NUP37 completely reversed all three LRP5 siRNA knockdown-induced inhibition in HepG2 cell proliferation, demonstrating that the LRP5 knockdown-induced inhibition on cell proliferation is specifically caused by destabilization of NUP37 (Fig. 4). 
A

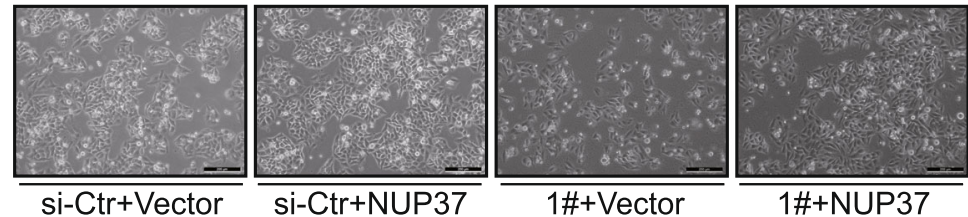

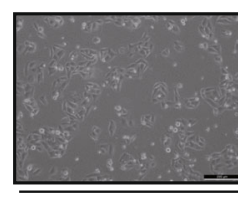

2\#+Vector

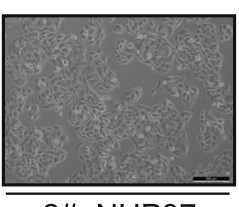

2\#+NUP37

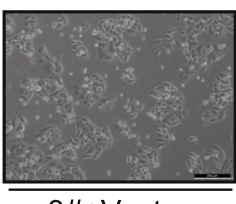

3\#+Vector

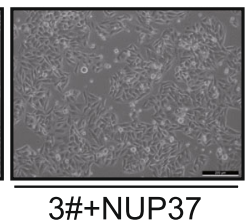

B

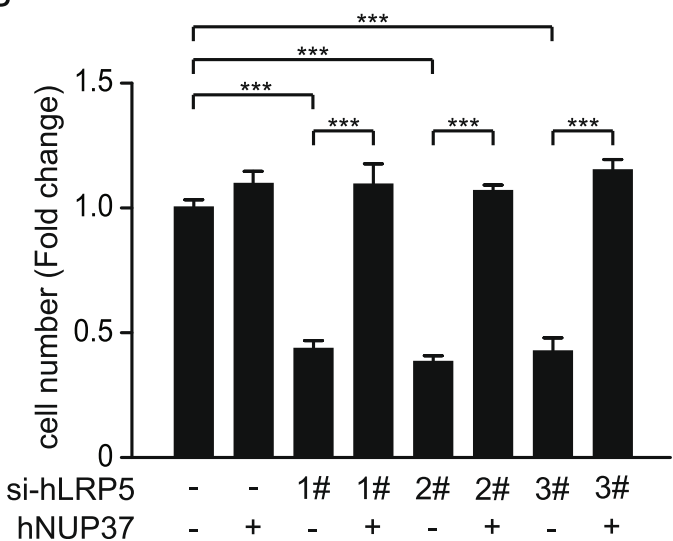

Fig. 4 NUP37 overexpression reverses LRP5 knockdown-induced inhibition of cell proliferation. a Representative image of HepG2 cells following over-expression of NUP37, which completely restored all three LRP5 siRNA (\#1, \#2, \#3) knockdown-induced inhibition of cell proliferation. $\mathbf{b}$ Quantification of total cell count, siRNA control cells were set as Fold change of $1 .{ }^{* *} P<0.001$

LRP5 overexpression restores NUP37 knockdown-induced downregulation of YAP pathway

As we demonstrated above, knockdown of LRP5 inhibited HepG2 cell proliferation via specific destabilization of NUP37. In order to clarify the underlying mechanisms, we used a YAP inhibitor CA3(CIL56), which significantly decreased HepG2 cell viability to $\sim 65 \%$, while knockdown of NUP37 using three different siRNAs all decreased HepG2 cell viability to $\sim 85 \%$ compared to scrambled siRNA-treated cells (Additional file 1: Figure S8). Of note, we did not observe a further reduction in proliferation when YAP was inhibited in NUP37 knockdown cells $(\sim 65 \%)$, suggesting that knockdown of NUP37 specifically affects proliferation via YAP/TEAD signaling. Therefore, it is reasonable to suggest that overexpression of LRP5 may be able to rescue NUP37 knockdown-induced downregulation of YAP/TEAD signaling. Indeed, knockdown of NUP37 with three distinct siRNAs all significantly decreased the expression levels of YAP/TEAD signaling target gene CTGF, but were restored by the overexpression of LRP5 (Fig. 5). Taken together, our results demonstrated that LRP5 bound to and modulated the stability of NUP37, thereby maintaining the dynamic integrity of NPCs and subsequently promoting cancer progression in HCC. Thus, LRP5 acts as a genuine regulator of YAP/TEAD signaling, suggesting a promising therapeutic target for preventing $\mathrm{HCC}$ proliferation.

\section{Discussion}

This is the first study which examined the physical and functional interaction between LRP5 and NUP37 in cancer. We demonstrated that knockdown of LRP5, but not LRP6 or $\beta$-catenin, markedly inhibited the proliferation of liver cancer HepG2 cells by destabilizing NUP37 and may result in the subsequent destruction of the NPC integrity. Moreover, overexpression of LRP5 restored NUP37 knockdown-induced downregulation of YAP/ TEAD signaling target gene CTGF, suggesting that LRP5 acts as a genuine regulator of YAP/TEAD signaling, 


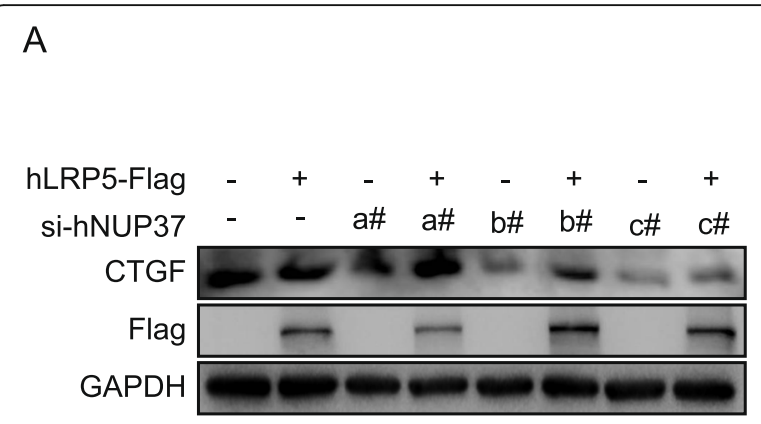

B

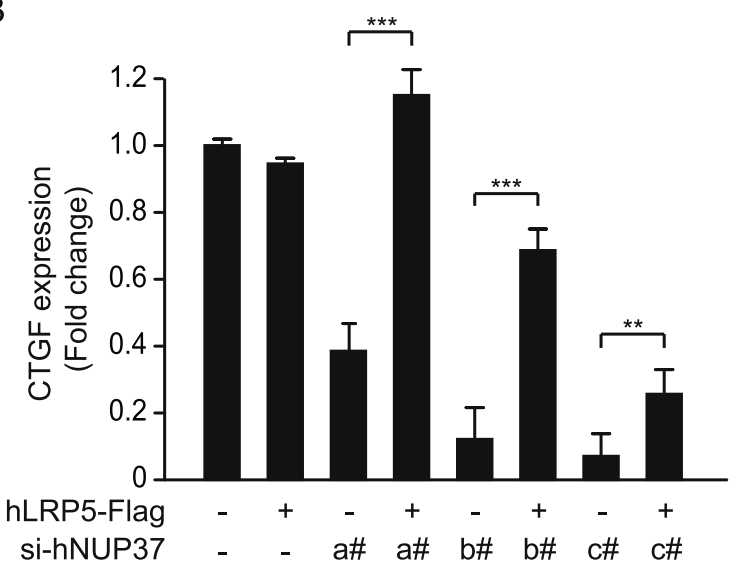

Fig. 5 LRP5 overexpression restores NUP37 knockdown induced down regulation of YAP pathway. a Western blot showing knockdown of NUP37 with three distinct siRNA (\#a, \#b, \#c) significantly decreases the expression level of YAP/TEAD signaling target gene CTGF, but restored by over-expression of $L R P 5, \mathbf{n}=3$. $\mathbf{b}$ Densitometry analysis normalized with the loading control, GAPDH

while also implicating LRP5 as a promising therapeutic target for preventing HCC proliferation.

Transport and translocation of signaling proteins between the cytoplasm and nucleus are tightly controlled by the NPC, a multi-protein channel located in a fusion pore between the outer and inner membranes of the nuclear envelope [11, 18-21]. The NPC is involved in the regulation of numerous cellular processes, such as gene expression and cell proliferation, but requires the synergistic cooperation between numerous components, and therefore, maintaining the integrity of the NPC is critical [10]. Our study revealed a previously unknown biological function of LRP5, whereby LRP5 binds to and stabilizes NUP37, one component of the outer rings of the NPC, which in turn maintains the integrity of the NPC. In contrast, LRP5 deficiency results in the destabilization of NUP37, and may lead to the destruction of the NPC integrity, which in turn causes dysregulation in the nuclear translocation of numerous signaling proteins, including those critically involved in the proliferation of liver cancer cells. Due to the fact that knockdown of NUP37 also significantly inhibited the proliferation of a separate liver cancer cell line Huh7, as well as non liver cancer cell line HEK-293, LRP5 may play a universal role in the modulation of NPC function via specific stabilization of NUP37. A recent study demonstrated that mutations in the genes encoding the outer ring components of the NPC, namely NUP107, NUP85, NUP133, and NUP160, were associated with the development of steroidresistant nephrotic syndrome (SRNS), indicating that maintaining the integrity of the NPC is crucial [22]. Due to the critical role of LRP5 in maintaining the integrity of the NPC, thereby promoting cellular proliferation, LRP5 may be involved in promoting cancer progression in HCC.

LRP5 is mostly expressed on the cell membrane but also had visible nuclear expression (Additional file 1: Figure S9), whereas NUP37 is almost exclusively expressed in the cell nucleus. Thus, it is likely that LRP5 can enter the cell nucleus and interact with NUP37 within the nucleus. However, because the endogenous nuclear expression of LRP5 was low, our current study was unable to detect an endogenous interaction between LRP5 and NUP37. Nevertheless, due to the fact that LRP5 strictly controlled HepG2 cell fate via regulating NUP37, it is possible that under certain disease conditions, the entry of LRP5 into the nucleus may allow the detection of endogenous interaction between LRP5 and NUP37, which requires further investigation. Destabilization of NUP37 induced by the absence of LRP5 may occur via protein degradation, which is a coordinated process that involves protein recognition, attachment of multiple ubiquitin molecules and subsequent digestion by the 26S proteasome [23-27]. The recognition domain of the ubiquitin-proteasome complex in NUP37 may be covered when it binds to LRP5 in the normal state, but may also be uncovered upon dysfunction of LRP5 under diseased conditions, which requires further investigation.

\section{Conclusions}

Our current study revealed a novel function of LRP5, which binds to and modulates the stability of NUP37 in a $\beta$ catenin-independent manner. Upregulation of NUP37 expression is critical for the progression of $\mathrm{HCC}$ via activation of YAP/TEAD signaling, where LRP5 may act as a genuine regulator of YAP/TEAD signaling via maintaining the integrity of the NPC. Our study implicates LRP5 as a promising therapeutic target for inhibiting liver cancer cell proliferation.

\section{Supplementary information}

Supplementary information accompanies this paper at https://doi.org/10. 1186/s12964-019-0495-3.

Additional file 1 : Figure S1. TOPflash assay showing the basal level of Wnt/ $\beta$-catenin pathway activation between HCC cell line HepG 2 cells and a non cancer cell line HEK293 cells. $n=3$. Figure S2. Tumor formation assay following injection of HepG2 cells in SCID/bg mice after transient transfection with control, LRP5/6, or $\beta$-catenin siRNAs. $n=4$. ${ }^{*} p<0.05$, 
n.s. no significance, compared to si-Ctr. Figure S3. TOPflash assay showing the individual roles of LRP5 and LRP6 in regulating Wnt/ $\beta$-catenin pathway. $n=3$. Figure $\mathbf{S 4}$. Western blots showing the knockdown efficiency of all three LRP5 siRNAs, as well as LRP6 and $\beta$-catenin siRNAs. $n=$ 3. GAPDH, loading control. Figure $\mathbf{S 5}$. Knockdown efficiency of all three NUP37 siRNAs as verified using real-time PCR assay (A), as well as western blot assay (B). $n=3$. GAPDH, loading control. *** $p<0.001$, compared to si-Ctr. Figure S6. MTT assay (A) and Photoimages (B) of Huh7 cell proliferation following knockdown of NUP37 or LRP5. $\mathrm{n}=3$. ${ }^{* * *} \mathrm{p}<0.001$, compared to si-Ctr. Figure S7. Photoimages and quantification of HEK-293 cell proliferation following knockdown of NUP37. $n=3$. ${ }^{* *} p<0.01$, *** $p<0.001$, compared to si-Ctr. Figure $\mathbf{S 8}$. MTT assay showing the decrease in cell proliferation following treatment with YAP inhibitor CA3(CIL56) in NUP37 knocked-down HepG2 cells. $n=3 .{ }^{* *} p<0.01,{ }^{* * *}$ $p<0.001$, compared to si-Ctr. Figure 9. Western blots showing the subcellular localization of LRP5 and NUP37. $n=3$.

\section{Abbreviations}

CTGF: Connective tissue growth factor; DKK1: Dickkopf-1; HCC: Hepatocellular carcinoma; IGFBP-4: Insulin-like growth factor binding protein-4; LRP5: Lowdensity lipoprotein-related receptor 5; LRP6: Low-density lipoprotein-related receptor 6; NPC: Nuclear pore complex; NUP37: Nucleoporin 37; siRNA: small interfering RNA; TEAD: TEA Domain transcription factor; YAP1: Yes-associated protein 1

\section{Acknowledgements}

Not applicable.

\section{Authors' contribution}

This manuscript was revised by D. W., who is a native English speaker. We thank J. C. and D. W. for assisting with illustration of figures. J. C. performed most of the experiments. E. M., H. Y. and J. P. contributed to new reagents/ analytic tools. D-N. R., D. W. and J. C. analyzed data. Y. F. and W. Z. assisted in instructing students and critical discussion. D-N. R. wrote the manuscript. DN. R. conceived and supervised the entire project. All authors read and approved the final manuscript.

\section{Funding}

This work was supported by National Natural Science Foundation of China (81672849, 81470395, 81600313, and 81673721), China Postdoctoral Science Foundation (2018 M642045) and Scientific Research Foundation for the Highlevel Talents, Fujian University of Traditional Chinese Medicine (X2019001talents).

\section{Availability of data and materials}

The datasets used and/or analysed during the current study are available from the corresponding author on reasonable request.

\section{Ethics approval and consent to participate}

Not applicable.

\section{Consent for publication}

Not applicable.

\section{Competing interests}

The authors declare that they have no competing interests.

\section{Author details}

'Department of Plastic and Burn Surgery, Ninth People's Hospital, Shanghai Jiaotong University, School of Medicine, 639 Zhi Zao Ju Road, Shanghai 200011, People's Republic of China. 'Fujian Key Laboratory of Integrative Medicine on Geriatric, Academy of Integrative Medicine, Fujian University of Traditional Chinese Medicine, 1 Qiuyang Road, Minhou Shangjie, Fuzhou 350122, Fujian, China. ${ }^{3}$ Clinical and Translational Research Center, Research Institute of Heart Failure Shanghai East Hospital, Key Laboratory of Arrhythmias of Ministry of Education, Tongji University School of Medicine, Shanghai, China.
Received: 6 September 2019 Accepted: 5 December 2019

Published online: 27 December 2019

\section{References}

1. Morin PJ, Sparks AB, Korinek V, et al. Activation of beta-catenin-Tcf signaling in colon cancer by mutations in beta-catenin or APC. Science. 1997;275: 1787-90.

2. MacDonald BT, He X. Frizzled and LRP5/6 receptors for Wnt/beta-catenin signaling. Cold Spring Harb Perspect Biol. 2012;4:a007880.

3. Hua $Y$, Yang $Y$, Li Q, et al. Oligomerization of frizzled and LRP5/6 protein initiates intracellular signaling for the canonical WNT/beta-catenin pathway. J Biol Chem. 2018;293:19710-24.

4. MacDonald BT, Tamai K, He X. Wnt/beta-catenin signaling: components, mechanisms, and diseases. Dev Cell. 2009;17:9-26.

5. He X, Semenov M, Tamai $K$, et al. LDL receptor-related proteins 5 and 6 in Wnt/beta-catenin signaling: arrows point the way. Development. 2004;131: 1663-77.

6. Tamai K, Semenov M, Kato Y, et al. LDL-receptor-related proteins in Wnt signal transduction. Nature. 2000;407:530-5

7. Ren DN, Chen J, Li Z, et al. LRP5/6 directly bind to frizzled and prevent frizzled-regulated tumour metastasis. Nat Commun. 2015;6:6906.

8. Wo D, Peng J, Ren DN, et al. Opposing roles of Wnt inhibitors IGFBP-4 and Dkk1 in cardiac ischemia by differential targeting of LRP5/6 and betacatenin. Circulation. 2016:134:1991-2007.

9. Chen Z, Li Y, Jiang G, et al. Knockdown of LRP6 activates Drp1 to inhibit survival of cardiomyocytes during glucose deprivation. Biomed Pharmacother. 2018;103:1408-14.

10. Chatel G, Fahrenkrog B. Dynamics and diverse functions of nuclear pore complex proteins. Nucleus. 2012;3:162-71.

11. Wente SR, Rout MP. The nuclear pore complex and nuclear transport. Cold Spring Harb Perspect Biol. 2010;2:a000562.

12. Harel A, Orjalo AV, Vincent T, et al. Removal of a single pore subcomplex results in vertebrate nuclei devoid of nuclear pores. Mol Cell. 2003;11:853-64.

13. Walther TC, Alves A, Pickersgill H, et al. The conserved Nup107-160 complex is critical for nuclear pore complex assembly. Cell. 2003;113:195-206.

14. Luo X, Liu Y, Feng W, et al. NUP37, a positive regulator of YAP/TEAD signaling, promotes the progression of hepatocellular carcinoma. Oncotarget. 2017:8:98004-13.

15. Uhlen $\mathrm{M}$, Zhang $\mathrm{C}$, Lee $\mathrm{S}$, et al. A pathology atlas of the human cancer transcriptome. Science. 2017;357:eaan2507. Patient liver cancer survival data were obtained online from The Human Protein Atlas website: https://www. proteinatlas.org/ENSG00000075188-NUP37/pathology/liver+cancer.

16. de La Coste A, Romagnolo B, Billuart $P$, et al. Somatic mutations of the beta-catenin gene are frequent in mouse and human hepatocellular carcinomas. Proc Natl Acad Sci U S A. 1998;95:8847-51.

17. Zhu W, Shiojima I, Ito Y, et al. IGFBP-4 is an inhibitor of canonical Wnt signalling required for cardiogenesis. Nature. 2008;454:345-9.

18. Sakiyama Y, Panatala R, Lim RYH. Structural dynamics of the nuclear pore complex. Semin Cell Dev Biol. 2017;68:27-33.

19. Beck $M$, Hurt $E$. The nuclear pore complex: understanding its function through structural insight. Nat Rev Mol Cell Biol. 2017;18:73-89.

20. Hampoelz B, Andres-Pons A, Kastritis $P$, et al. Structure and assembly of the nuclear pore complex. Annu Rev Biophys. 2019;48:515-36.

21. Lin $\mathrm{DH}$, Hoelz A. The structure of the nuclear pore complex (an update) Annu Rev Biochem. 2019;88:725-83.

22. Braun DA, Lovric S, Schapiro D, et al. Mutations in multiple components of the nuclear pore complex cause nephrotic syndrome. J Clin Invest. 2018; 128:4313-28.

23. Nandi D, Tahiliani P, Kumar A, et al. The ubiquitin-proteasome system. J Biosci. 2006;31:137-55

24. Marcinkiewicz K, Substrate-engaged M. 26S proteasome. Nat Struct Mol Biol. 2018;25:1069.

25. Bard JAM, Goodall EA, Greene ER, et al. Structure and function of the $26 \mathrm{~S}$ proteasome. Annu Rev Biochem. 2018:87:697-724.

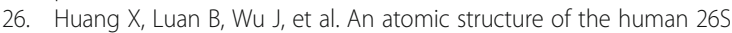
proteasome. Nat Struct Mol Biol. 2016:23:778-85.

27. Collins GA, Goldberg AL. The logic of the 265 proteasome. Cell. 2017;169:792-806.

\section{Publisher's Note}

Springer Nature remains neutral with regard to jurisdictional claims in published maps and institutional affiliations. 